\title{
Generation of nonclassical microwave states using an artificial atom in 1D open space
}

\author{
Io-Chun Hoi, Tauno Palomaki, Göran Johansson, Joel Lindkvist, Per Delsing \& C.M.Wilson* \\ Department of Microtechnology and Nanoscience (MC2), \\ Chalmers University of Technology, Göteborg, Sweden.
}

(Dated: November 8, 2018)

\begin{abstract}
We have embedded an artificial atom, a superconducting transmon qubit, in a 1D open space and investigated the scattering properties of an incident microwave coherent state. By studying the statistics of the reflected and transmitted fields, we demonstrate that the scattered states can be nonclassical. In particular, by measuring the second-order correlation function, $g^{(2)}$, we show photon antibunching in the reflected field and superbunching in the transmitted field. We also compare the elastically and inelastically scattered fields using both phase-sensitive and phase-insensitive measurements.
\end{abstract}

PACS numbers: 42.50.Gy,03.67.Hk,42.50.Ar,85.25.Cp

A single atom interacting with propagating electromagnetic fields in open space is a fundamental system of quantum optics. Strong coupling between a single artificial atom and resonant propagating fields has recently been achieved in a 1D system [1, 2], experimentally demonstrating nearly perfect extinction of the forward propagating fields [2]. However, this extinction can be explained by classical theory: a classical point-like oscillating dipole perfectly reflects resonant incident fields [3]. In this Letter, we demonstrate the quantum nature of the transmitted and reflected fields generated from our artificial atom in 1D open space by using a resonant coherent state as the incident field. In particular, by measuring the statistics of the fields we show that the reflected field is antibunched [4, 5] while still maintaining first-order coherence. Moreover, we observe superbunching statistics in the transmitted fields [4].

To understand how our artificial atom generates antibunched and superbunched states, it is helpful to consider the incident coherent state in the photon number basis. For a low power incident field with less than 0.5 average photons per lifetime of our atom, we can safely approximate the coherent field using only the first three photon eigenstates. If we consider a one-photon incident state, the atom reflects it, leading to antibunching statistics in the reflected field. Together with the zerophoton state the reflected field still maintains first-order coherence. For a two-photon incident state, since the atom is not able to scatter more than one photon at a time, the pair has a much higher probability of transmission, leading to superbunching statistics in transmission [4, 6]. In this sense, our single artificial atom acts as a photon-number filter, which extracts the one-photon number state from a coherent state. This represents a novel way to generate photon correlations and nonclassical states in the microwave compared to other recent work 7 11].

Our system consists of a superconducting transmon qubit [12], strongly coupled to a $1 \mathrm{D}$ transmission line in a coplanar waveguide configuration(see Fig. 1A). The

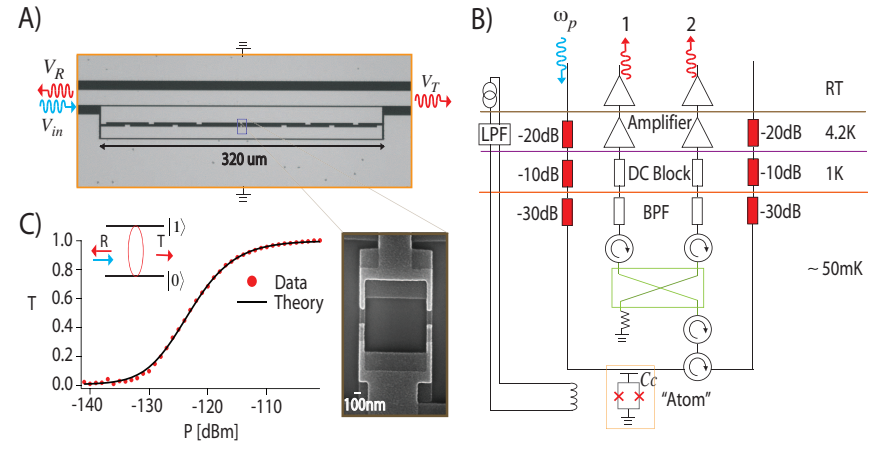

FIG. 1. (A) A micrograph of our artificial atom, a superconducting transmon qubit embedded in a $1 \mathrm{D}$ open transmission line. (Zoom In) Scanning-electron micrograph of the SQUID loop of the transmon. (B) Schematic setup for measurement of the second-order correlation function. This setup enables us to do Hanbury Brown-Twiss measurements between output ports 1 and 2. Depending on the choice of input port, we can measure $g^{(2)}$ of the reflected/transmitted field. (C) Transmittance on resonance as a function of incident power. (Inset) A weak, resonant coherent state is reflected by the atom.

ground state $|0\rangle$ and first excited state $|1\rangle$ have a transition energy $\hbar \omega_{01}$. The relaxation rate of the atom is dominated by an intentionally strong coupling to the $\mathrm{Z}$ $=50 \Omega$ transmission line through the coupling capacitor $C_{c}$, defined in Fig. 1B.

The electromagnetic field in the transmission line is described by an incoming voltage wave $V_{i n}$, a reflected wave $V_{R}$ and a transmitted wave $V_{T}$. In Fig.1A, the transmittance is defined as $T=\left|V_{T} / V_{i n}\right|^{2}$. For a weak coherent drive on resonance with the atom, we expect to see full reflection of the incident signal [4, 13]. This can be understood in terms of interference between the incident wave and wave scattered from the atom, which destructively interferes in transmission and constructively interferes in reflection [4, 13]. In the sample measured here, we achieved extinction of more than $99 \%$ in transmittance, as shown in Fig. 1C. By measuring the transmission coef- 
ficient as a function of probe frequency and probe power $P$, we extract $\omega_{01} / 2 \pi=5.12 \mathrm{GHz}, \Gamma_{10} / 2 \pi=41 \mathrm{MHz}$, $\Gamma_{\phi} / 2 \pi=1 \mathrm{MHz}$. The relaxation rate $\Gamma_{10}$ is dominated by the coupling to the line and is much greater than the pure dephasing $\Gamma_{\phi}$ in our system. We define an average number of photons per interaction time $2 \pi / \Gamma_{10}$ as $N \equiv 2 \pi P /\left(\hbar \omega_{p} \Gamma_{10}\right) . \quad N=1$ for a power of $-128 \mathrm{dBm}$.

The resonant electromagnetic field reflected from or transmitted through the atom (depending on the choice of input port, see Fig 1B) is fed through two circulators to a commercial $90^{\circ}$ hybrid coupler, with its other input terminated with $50 \Omega$. The hybrid coupler effectively acts as a microwave beam splitter. Ideally, the signal coming into the other input of the hybrid coupler should be vacuum. The two outputs of the beam splitter are sent to two nominally identical microwave HEMT amplifiers at $4.2 \mathrm{~K}$ that have system noise temperatures of $7 \mathrm{~K}$. We make the essential assumption in our analysis that the noise added by the two amplifiers is uncorrelated. After further amplification, the two outputs are fed into a pair of vector digitizers, which capture the voltage amplitude. We then can choose to digitally filter the voltage data to a desired bandwidth, BW. This setup enables us to perform Hanbury Brown-Twiss (HBT) [14 18] measurements using linear quadrature detectors, i.e., amplifiers.

Second-order correlation measurements provide a method to characterize microwave states generated in our system. In particular, they provide a statistical tool to show that the scattered light is nonclassical. For reference, we first considered a thermal state and a coherent state. The thermal state [19] was generated by simply amplifying the noise of a $50 \Omega$ resistor through room temperature amplifiers before sending the signal down through the transmission line in our set-up, with the qubit off-resonance. The two digitizers were set to a sampling frequency of $10^{8}$ samples/sec and then the voltages were digitally filtered separately. Finally, we determine the power-power correlations as a function of delay time between the two outputs.

The second order correlation function can be expressed as

$$
g^{(2)}(\tau)=1+\frac{\left\langle\Delta P_{1}(t) \Delta P_{2}(t+\tau)\right\rangle}{\left[\left\langle P_{1}(t)\right\rangle-\left\langle P_{1, N}(t)\right\rangle\right]\left[\left\langle P_{2}(t)\right\rangle-\left\langle P_{2, N}(t)\right\rangle\right]},
$$

where $\tau$ is the delay time between the two digitizers, $P_{1}, P_{2}$ are the output powers in ports 1 and 2, respectively. $P_{1, N}, P_{2, N}$ are the amplifier noise in ports 1 and 2 , respectively, when the incident source is off. Therefore, $\left[\left\langle P_{i}(t)\right\rangle-\left\langle P_{i, N}(t)\right\rangle\right]$ represents the net power of the field from output port $\mathrm{i}$, where $i=1,2 .\left\langle\Delta P_{1} \Delta P_{2}\right\rangle$ is the covariance of the output powers in port 1 and 2 , defined as $\left\langle\left(P_{1}-\left\langle P_{1}\right\rangle\right)\left(P_{2}-\left\langle P_{2}\right\rangle\right)\right\rangle$. The following assumptions were made: (1) the amplifier noise originating from the two independent detection chains is uncorrelated, and (2) the $50 \Omega$ terminator is in its ground state $(5 \mathrm{GHz}$ photons at $\sim 50 \mathrm{mK})$. In Fig. $2 \mathrm{~A}$, we show $g^{(2)}$ as a func- tion of delay time $\tau$, for a thermal state with two different filter bandwidths, and also for a coherent state. For thermal states, $g^{(2)}(0)=2$ regardless of the filter bandwidth BW. The width of $g^{(2)}(\tau)$ for the thermal state is determined by the filter function. For the filter used $g^{(2)}(\tau)=1+e^{-2 \pi B W|\tau|}$, the solid curves of the thermal state in Fig. 2A show this equation with no free fitting parameters. We had a trigger jitter of \pm 1 sample between the two digitizers. To minimize the effect of this trigger jitter, we oversample and then digitally filter (average) the data in all the $g^{(2)}$ measurements. For a coherent state, we expect $g^{(2)}(\tau)=1$. This is indeed what we find if our atom is off-resonance from our applied coherent source.

After making these initial measurements, we made second order correlation measurements to study the field transmitted through our atom. We applied an onresonance microwave drive and measured $g^{(2)}(\tau)$ in transmission for different incident powers as shown in Fig. 2B. Here the sampling frequency was again set to $10^{8}$ samples/s with $\mathrm{BW}=55 \mathrm{MHz}$. At the lowest power $(P=-129 \mathrm{dBm}, N=0.8)$ that we can readily measure, we see superbunching of the photons as expected [4], with $g^{(2)}(\tau=0)=2.31 \pm 0.09>2$. Superbunching occurs because the one-photon state of the incident field has been selectively filtered and reflected while the twophoton state is more likely transmitted (the three-photon components and higher are negligible). This transmitted state generated from our qubit is thus bunched even more than a thermal state. For high powers, where $N \gg 1$, we find $g^{(2)}(\tau)=1$ as expected, because most of the coherent signal then passes through the transmission line without interacting with the atom owing to saturation of the atomic response. The correlation once again looks like a coherent state. For all measurements shown here we find, $g^{(2)}(\infty)=1$, as expected. In the right inset of Fig. 2B, we plot $g^{(2)}(0)$ as a function of incident power, and clearly see the bunching behavior decrease as the incident power increases. For comparison, we also plot the coherent state and thermal state.

In Fig. 2C, we show the measured $g^{(2)}(\tau)$ of the reflected field from our atom. At low powers, where $N \ll 1$, we clearly observe antibunching of the field [4]. Each trace here was collected, computed and averaged over 17 hours, corresponding to $2.4 \times 10^{11}$ measured quadrature field samples (2 Tbyte of data). The antibunching behavior at the lowest power $(P=-132 \mathrm{dBm}, N=0.4)$, $g^{(2)}(0)=0.51 \pm 0.05$, reveals the quantum nature of the field. Ideally, we would find $g^{(2)}(0)=0$ as the atom can only absorb and emit one photon at a time. The non-zero $g^{(2)}(0)$ we measured is most likely from the combination of trigger jitter between the two digitizers and the filter bandwidth (here $55 \mathrm{MHz}$ ). In Fig. 2D, we clearly see how the antibunching dip depends on bandwidth. For small $\mathrm{BW}$, where $\mathrm{BW} \ll \Gamma_{01}$, the antibunching dip vanishes entirely. With a small BW or long sampling time, 

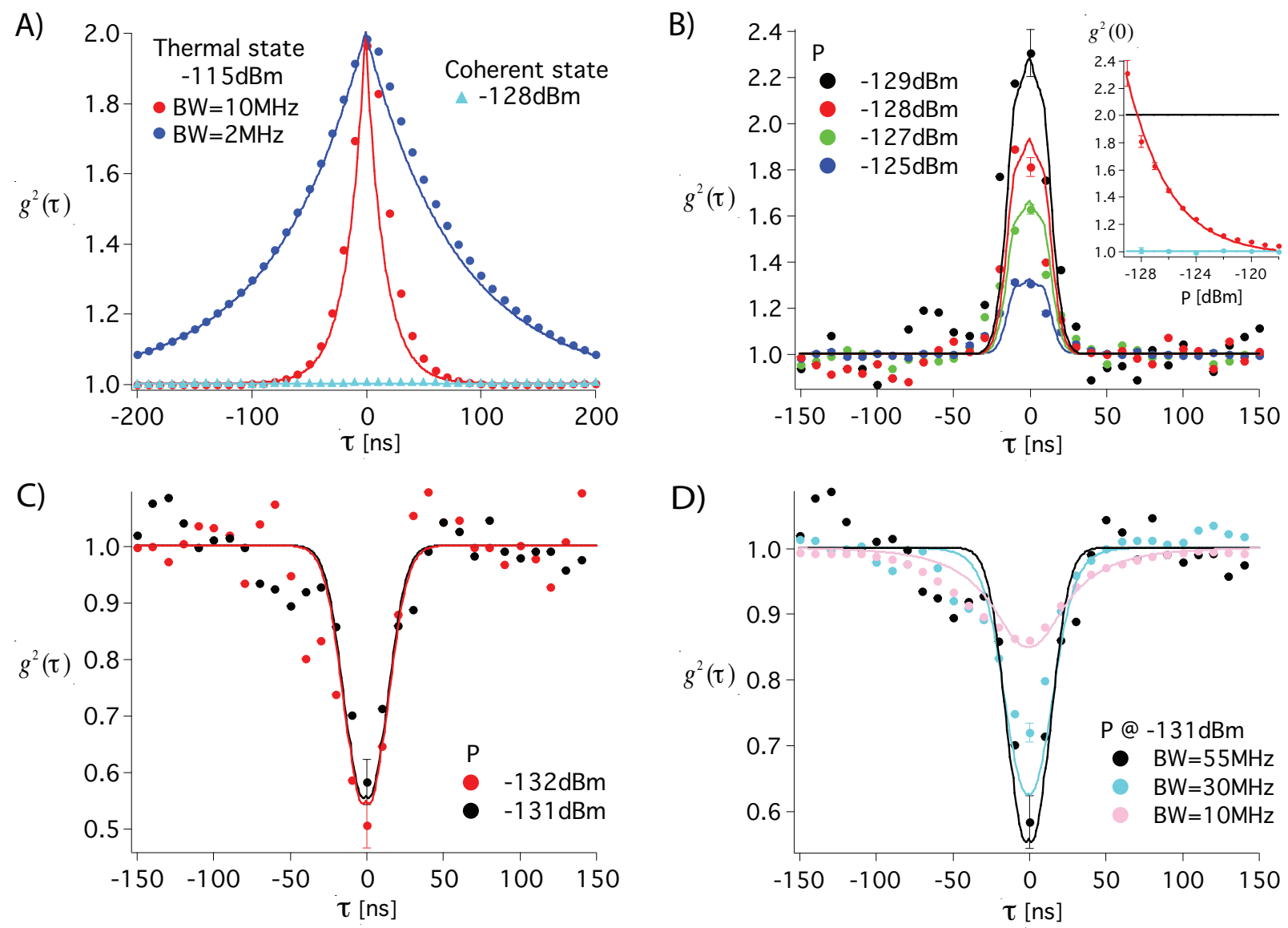

FIG. 2. Second-order correlation function of a thermal state, a coherent state and the states generated by the artificial atom. A) $g^{(2)}$ of a thermal state and a coherent state as a function of delay time $\tau$. B) $g^{(2)}$ of the resonant transmitted microwaves as a function of delay time for four different incident powers. Inset: $g^{(2)}(0)$ as a function of incident power. For comparison, the result for a thermal state and a coherent state are also plotted. We see that the transmitted field statistics(red curve) approaches that of a coherent field at high incident power, as expected. For a coherent state $g^{(2)}(0)=1$ is independent of incident power (blue). C) $g^{(2)}$ of a resonant reflected field as a function of delay time for two different incident powers. The antibunching behavior reveals the quantum nature of the field. The curves shown here had a digital filter with a $55 \mathrm{MHz}$ bandwidth applied to each detector. D) $g^{(2)}$ of a resonant reflected field as a function of delay time at $-131 \mathrm{dBm}$ incident power for different filter bandwidths. As the bandwidth decreases, the antibunching dip vanishes. The solid curves in B), C) and D) are the theory curves, including trigger jitter model (see text). We extract a temperature of $50 \mathrm{mK}$ from these fits, with no additional free fitting parameters. The peculiar feature of $g^{(2)}$ around zero in the theory curves in B) is due to the trigger jitter model. The error bar indicated for each data set is the same for all the points.

the time dynamics of antibunching cannot be resolved. In other words, within that time interval, the atom has had time to absorb and emit many photons.

To obtain the theoretical results in Fig. 2 B, C, and $\mathrm{D}$, we use a master equation formalism. The digital filter is modeled by a single-mode resonator. A master equation describing both the transmon and the resonator is derived using the formalism of cascaded quantum systems. To model the effect of the trigger jitter, the value of $g^{(2)}(\tau)$ at each point is replaced by the average value of $g^{(2)}(\tau), g^{(2)}(\tau-10 \mathrm{~ns})$ and $g^{(2)}(\tau+10 \mathrm{~ns})$.

Our artifical atom selectively filters out the Fock state $n=1$ from the input coherent state. As a result, the reflected and transmitted field display antibunched and superbunched statistics, respectively. Thus, the qubit acts as a passive photon-number filter, converting a coherent microwave state to a nonclassical one, with high production rate.

While the scattered field requires a purely quantum description, it can still maintain first-order coherence similar to a classical field, as shown below. We can define the first-order correlation function in steady state as $g^{(1)}=\langle V\rangle^{2} /\left\langle V^{2}\right\rangle$. First-order coherence then refers to $g^{(1)}=1$. As expected, for a thermal source, this function is 0 and for a coherent state it is 1 .

The first-order coherence properties of the scattered resonance field strongly depend on the Rabi frequency $\Omega_{p}$ and the relaxation rate $\Gamma_{10}$ of the atom. The Rabi 

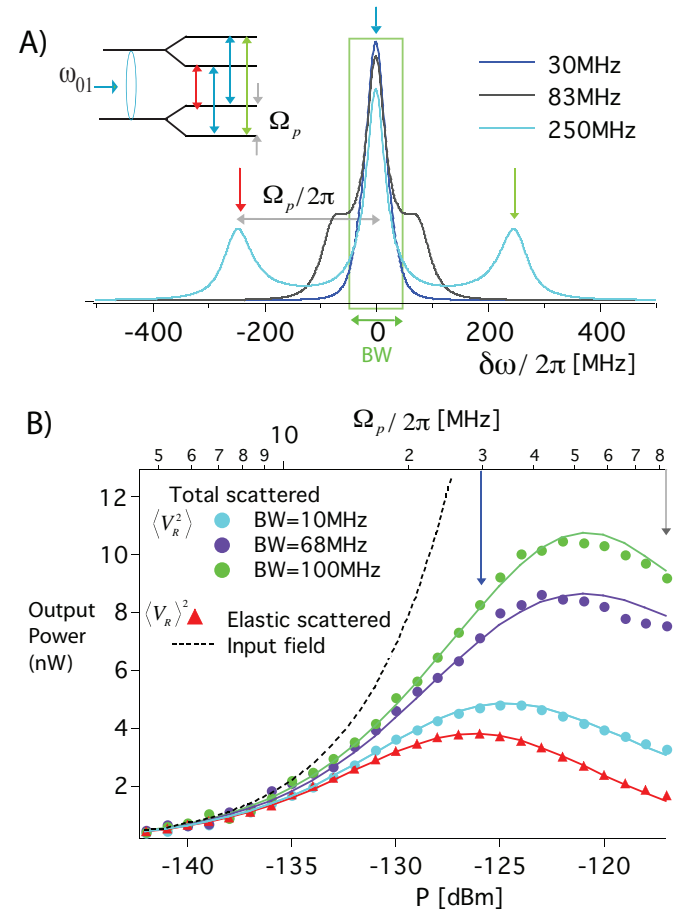

FIG. 3. Elastic vs. inelastic scattering from the artificial atom. A) A microwave pump is applied at $\omega_{01}$. As the power of the $\omega_{01}$ pump increases, the Mollow triplet appears in the spectrum with peak separation equal to the Rabi frequency $\Omega_{p}$. (inset) Dressed state picture of the energy levels. B) The coherently/elastically reflected power (phase-sensitive average, red curve) or total reflected power (phase-insensitive average, green, grey and blue curves) as a function of resonant incident powers for different bandwidths (BW). The total power reflected is the sum of both the elastic and inelastic fields, after we subtract off the amplifier noise power. Solid curves are the theory fits to experimental data. The black dash curve shows the input power for comparison. At low powers, $N<<1$, we observed that the reflected field is mostly first-order coherent: the elastically reflected power is the same as the total reflected power. At high powers, $N>1$, more and more photons are inelastically scattered as the Mollow triplet begins to emerge. The wider the measurement bandwidth, the more of the Mollow triplet we capture. Note that the output power includes the $79 \mathrm{~dB}$ gain of the amplifiers.

frequency $\Omega_{p}$ is linearly proportional to the amplitude of the drive, $\Omega_{p} \propto \sqrt{P}[2]$. For $\Omega_{p} \ll \Gamma_{10}$, we expect the scattered fields to be mostly coherent to first order, with a spectrum well described by a delta function [5] at $\omega_{01}$. For $\Omega_{p}>\Gamma_{10}$, the scattered fields contain three additional inelastic Lorentzians (known as the Mollow Triplet [20]) centered on the frequencies $\omega=\omega_{01}, \omega=$ $\omega_{01}+\Omega_{p}$ and $\omega=\omega_{01}-\Omega_{p}$, indicated in the theory plot Fig. 3A.

We send a single tone at $\omega_{01}$ and measure these scattered (reflected) fields from only one of the output ports, as shown in Fig. 3B. Note that we see the same behavior from both outputs. We use a phase-sensitive average $\langle V\rangle^{2}$ to capture the elastic (coherent) component of the scattered field. For the total scattered field, the sum of the elastically and inelastically scattered fields, we use a phase-insensitive average $\left\langle V^{2}\right\rangle$. The amount of the inelastic field that we capture depends on the bandwidth (BW) of our measurement, as indicated in Fig. 3A. The solid curves are the theory fits, using the model in Fig. 3A (integrating the Mollow triplet), with no free fitting parameters (using the same parameters extracted before). As expected, at low incident power, the total scattered field is roughly equal to the elastically scattered (coherent) field. This indicates that the field is first-order coherent with $g^{(1)} \simeq 1$. We note that this is also the regime where antibunching is observed. At high incident fields, where $\Omega_{p}>\Gamma_{10}$, the main contribution to the total field is from inelastic scattering.

In conclusion, we investigated the scattering properties of a single artificial atom in a $1 \mathrm{D}$ open space by measuring the first, second and fourth moments of the voltage field. We verified the quantum nature of the scattered field using the second-order correlation function, while also showing that the field maintained first-order coherence. In fact, the whole process leads to a redistribution of the photon number state [6].

We plan to investigate applications of this phenomenon, such as generating single-photon states on demand. This system may offer advantages over placing an artificial atom in a cavity [21 23], for instance. In our system, regardless of the strength of the input pulse only a single photon state is reflected. This eliminates the need for precise Rabi pulses to excite the atom as in a cavity-based photon source. Furthermore, our generated single photons can maintain the same envelope as the incident coherent state with a wide bandwidth limited by the atom relaxation rate. For a cavity-based photon source, the bandwidth is limited by the cavity width and subject to the problem of stochastic release by the cavity.

We acknowledge financial support from the Swedish Research Council, the Wallenberg foundation, SOLID and from the European Research Council. We would also like to acknowledge G. Milburn and T. Stace for fruitful discussions.

* chris.wilson@chalmers.se

[1] O. Astafiev, A. M. Zagoskin, Jr. A. A. Abdumalikov, Y. A. Pashkin, T. Yamamoto, K. Inomata, Y. Nakamura, and J. S. Tsai, Science 327, 840 (2010)

[2] I. C. Hoi, C. M. Wilson, G. Johansson, T. Palomaki, B. Peropadre, and P. Delsing, Phys. Rev. Lett. 107, 073601 (2011)

[3] G. Zumofen, N. M. Mojarad, V. Sandoghdar, and M. Agio, Physical Review Letters 101, 180404 (2008)

[4] D. E. Chang, A. S. Sorensen, E. A. Demler, and M. D. Lukin, Nature Physics 3, 807 (2007) 
[5] R. Loudon, The Quantum Theory of Light (Oxford science publications, 2010)

[6] H. Zheng, D. J. Gauthier, and H. U. Baranger, Physical Review A 82, 063816 (2010)

[7] C. M. Wilson, G. Johansson, A. Pourkabirian, M. Simoen, J. R. Johansson, T. Duty, F. Nori, and P. Delsing, Nature 479, 376 (2011)

[8] C. Eichler, D. Bozyigit, C. Lang, M. Baur, L. Steffen, J. M. Fink, S. Filipp, and A. Wallraff, Physical Review Letters 107, 113601 (2011)

[9] C. Eichler, D. Bozyigit, C. Lang, L. Steffen, J. Fink, and A. Wallraff, Physical Review Letters 106, 220503 (2011)

[10] B. Reulet, J. Senzier, and D. E. Prober, Physical Review Letters 91, 196601 (2003)

[11] F. Mallet, M. A. Castellanos-Beltran, H. S. Ku, S. Glancy, E. Knill, K. D. Irwin, G. C. Hilton, L. R. Vale, and K. W. Lehnert, Physical Review Letters 106, 220502 (2011)

[12] J. Koch, T. M. Yu, J. Gambetta, A. A. Houck, D. I. Schuster, J. Majer, A. Blais, M. H. Devoret, S. M. Girvin, and R. J. Schoelkopf, Physical Review A 76, 042319 (2007)

[13] J. T. Shen and S. H. Fan, Physical Review Letters 95, 213001 (2005)

[14] R. H. Brown and R. Q. Twiss, Nature 177, 27 (1956)

[15] J. Gabelli, L.-H. Reydellet, G. Fève, J.-M. Berroir,
B. Plaçais, P. Roche, and D. C. Glattli, Phys. Rev. Lett. 93, 056801 (2004)

[16] M. P. da Silva, D. Bozyigit, A. Wallraff, and A. Blais, Phys. Rev. A 82, 043804 (2010)

[17] N. B. Grosse, T. Symul, M. Stobińska, T. C. Ralph, and P. K. Lam, Phys. Rev. Lett. 98, 153603 (2007)

[18] E. P. Menzel, F. Deppe, M. Mariantoni, M. Á. Araque Caballero, A. Baust, T. Niemczyk, E. Hoffmann, A. Marx, E. Solano, and R. Gross, Phys. Rev. Lett. 105, 100401 (2010)

[19] Technically, it is a chaotic state.

[20] B. R. Mollow, Physical Review 188, 1969 (1969)

[21] D. Bozyigit, C. Lang, L. Steffen, J. M. Fink, C. Eichler, M. Baur, R. Bianchetti, P. J. Leek, S. Filipp, M. P. da Silva, A. Blais, and A. Wallraff, Nature Physics 7, 154 (2011)

[22] C. Lang, D. Bozyigit, C. Eichler, L. Steffen, J. M. Fink, Jr. A. A. Abdumalikov, M. Baur, S. Filipp, M. P. da Silva, A. Blais, and A. Wallraff, Phys. Rev. Lett. 106, 243601 (2011)

[23] A. A. Houck, D. I. Schuster, J. M. Gambetta, J. A. Schreier, B. R. Johnson, J. M. Chow, L. Frunzio, J. Majer, M. H. Devoret, S. M. Girvin, and R. J. Schoelkopf, Nature 449, 328 (2007) 